\title{
ColistinDose, a Mobile App for Determining Intravenous Dosage Regimens of Colistimethate in Critically III Adult Patients: Clinician-Centered Design and Development Study
}

Xueliang $\mathrm{Hua}^{1 *}$, BSc; Chen $\mathrm{Li}^{2,3,4^{*}}, \mathrm{PhD}$; Jason M Pogue ${ }^{5}$, PharmD; Varun S Sharma ${ }^{4}$, MSc; Ilias Karaiskos ${ }^{6}, \mathrm{MD}_{\text {, }}$ PhD; Keith S Kaye ${ }^{7}$, MD, MPH; Brian T Tsuji ${ }^{8,9}$, PharmD; Phillip J Bergen ${ }^{3,10}$, PhD; Yan Zhu ${ }^{3,10}$, PhD; Jiangning Song ${ }^{2,3,11}, \mathrm{PhD} ; \mathrm{Jian} \mathrm{Li}{ }^{3,10}, \mathrm{PhD}$

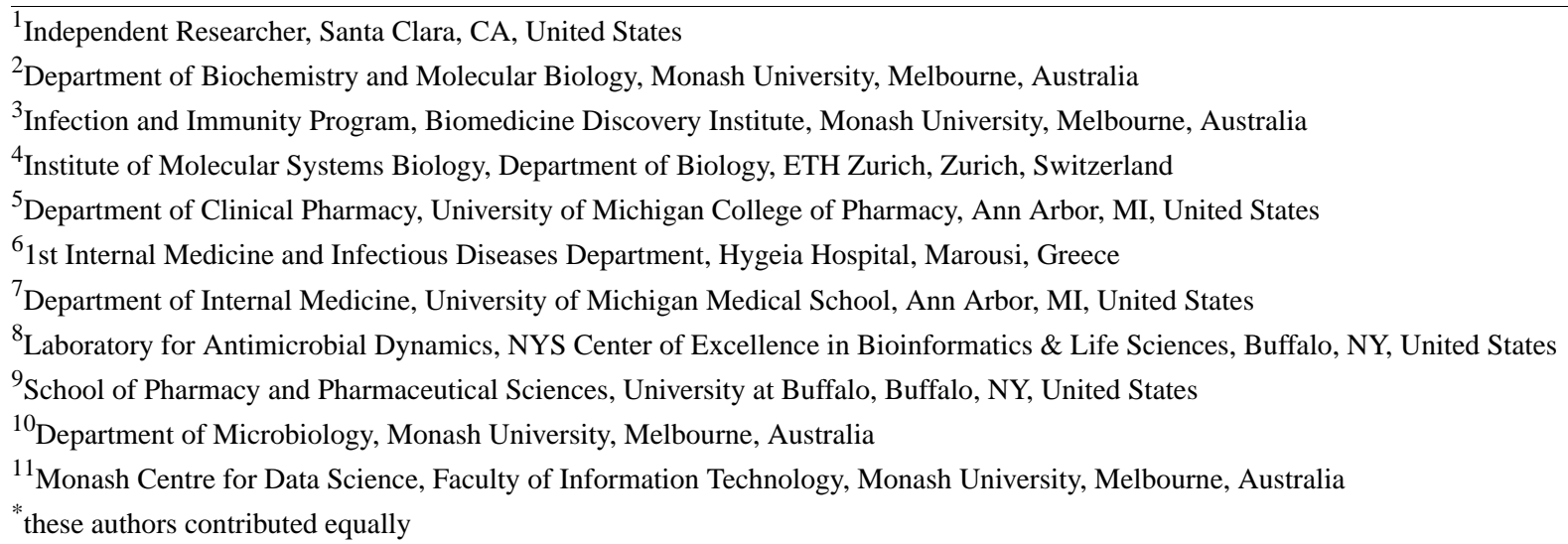

\section{Corresponding Author:}

Jian Li, PhD

Department of Microbiology

Monash University

19 Innovation Walk, Monash University

Melbourne, VIC 3800

Australia

Phone: 61399039702

Email: jian.li@monash.edu

\section{Related Article:}

This is a corrected version. See correction statement in: https://mhealth.jmir.org/2020/12/e26593/

\section{Abstract}

Background: Determining a suitable dose of intravenous colistimethate is challenging because of complicated pharmacokinetics, confusing terminology, and the potential for renal toxicity. Only recently have reliable pharmacokinetic/pharmacodynamic data and dosing recommendations for intravenous colistimethate become available.

Objective: The aim of this work was to develop a clinician-friendly, easy-to-use mobile app incorporating up-to-date dosing recommendations for intravenous colistimethate in critically ill adult patients.

Methods: Swift programming language and common libraries were used for the development of an app, ColistinDose, on the iPhone operating system (iOS; Apple Inc). The compatibility among different iOS versions and mobile devices was validated. Dosing calculations were based on equations developed in our recent population pharmacokinetic study. Recommended doses generated by the app were validated by comparison against doses calculated manually using the appropriate equations.

Results: ColistinDose provides 3 major functionalities, namely (1) calculation of a loading dose, (2) calculation of a daily dose based on the renal function of the patient (including differing types of renal replacement therapies), and (3) retrieval of historical calculation results. It is freely available at the Apple App Store for iOS (version 9 and above). Calculated doses accurately reflected doses recommended in patients with varying degrees of renal function based on the published equations. ColistinDose performs calculations on a local mobile device (iPhone or iPad) without the need for an internet connection. 
Conclusions: With its user-friendly interface, ColistinDose provides an accurate and easy-to-use tool for clinicians to calculate dosage regimens of intravenous colistimethate in critically ill patients with varying degrees of renal function. It has significant potential to avoid the prescribing errors and patient safety issues that currently confound the clinical use of colistimethate, thereby optimizing patient treatment.

(JMIR Mhealth Uhealth 2020;8(12):e20525) doi: $\underline{10.2196 / 20525}$

\section{KEYWORDS}

ColistinDose; colistimethate; colistin methanesulfonate; colistin; polymyxins; mobile app; renal function; renal replacement therapy; intermittent hemodialysis; sustained low-efficiency dialysis; continuous renal replacement therapy

\section{Introduction}

As the drug discovery pipeline for new antibiotics has dwindled $[1,2]$, multidrug-resistant gram-negative pathogens have become a serious global health threat [3]. Colistin (ie, polymyxin E) has increasingly been used as a "last-line" therapy to treat infections caused by gram-negative "superbugs" unresponsive to other agents [4,5]. In clinical settings, colistin is most commonly available as an inactive prodrug, colistimethate (also known as colistin methanesulfonate [CMS]), for intravenous and inhalational administration [6]. Unfortunately, nephrotoxicity following intravenous administration of polymyxins (colistin or polymyxin B) can occur in up to $60 \%$ of patients and is the major dose-limiting factor [7-9]. Owing to earlier difficulties in determining concentrations of colistimethate and formed colistin, only relatively recently have studies reliably investigated the pharmacokinetics (PK) of colistimethate and formed colistin in patients [10-18]. These studies have revealed the extremely complicated PK of colistimethate and formed colistin, which in turn has made determining appropriate dosing regimens for intravenous colistimethate very challenging. The complex PK of both the prodrug and formed colistin in patients extend to those with different renal functions and those on different renal replacement therapies (RRT) given that the apparent clearance of colistin is dependent on renal function [10]. Adding to this complexity is that the dose units of colistimethate are expressed differently in different parts of the world, namely as either colistin base activity (CBA) or number of international units (IU) (approximately $33.3 \mathrm{mg}$ of $\mathrm{CBA}=1$ million IU=approximately $80 \mathrm{mg}$ of $\mathrm{CMS}$ ); these different expressions are known to have caused prescribing errors and patient safety issues, and substantially confound the clinical use of colistimethate [19]. Selecting an optimal dose of colistimethate in critically ill patients is thus a difficult process with serious consequences for both underdosing (treatment failure and development of resistance) and overdosing (toxicity).

Mobile devices have become commonplace in health care settings. Professional mobile apps have created paradigm shifts in modern medicine in a number of areas including information storage and access, patient management and monitoring, clinical decision making, and clinical practice transformation [20].
Given the role of colistimethate as one of the most important last-line therapies for multidrug-resistant gram-negative bacteria and the difficulties associated with accurately determining an appropriate dose across many patient groups (including those on RRT), a user-friendly smartphone app utilizing the latest clinical pharmacological findings to determine optimal, personalized dosing regimens of intravenous colistimethate in patients would provide valuable assistance to clinicians.

We recently published the most comprehensive population PK model to date with dosing recommendations based on data from a total of 214 critically ill patients that included 29 patients on different forms of RRT [11]. To the best of our knowledge, there are no apps and only two online tools available for the calculation of colistimethate dosage regimens: a colistin dosing calculator [21] and a colistin calculator [22]. Neither tool utilizes the latest population PK model [11] for calculating the dosage regimens of intravenous colistimethate. This study aimed to develop an app, ColistinDose, for the iPhone operating system (iOS; Apple Inc) to allow clinicians to accurately and conveniently determine the dosing regimens of intravenous colistimethate on a local iPhone or iPad. Our app provides clinicians with an easy-to-use tool for determining optimal dosing regimens of intravenous colistimethate in adult patients with varying degrees of renal function directly at the bedside.

\section{Methods}

\section{Development of the ColistinDose Smartphone App}

Xcode and Interface Builder (Apple Inc) were employed to build the app's human interaction interfaces. Several open-source libraries (including Charts, Former, Persei, and Realm; please refer to the Multimedia Appendix 1 and the Acknowledgments section in the app for detailed information) were also utilized to implement relevant user interface elements, animation, and, in particular, local data storage. The overall design of the workflow of ColistinDose is shown in Figure 1. We employed the Swift programming language and common libraries for iOS to ensure that ColistinDose was functional on different screen sizes and versions of the operating system on iPhones and iPads. 
Figure 1. The workflow of ColistinDose. RRT: renal replacement therapy.

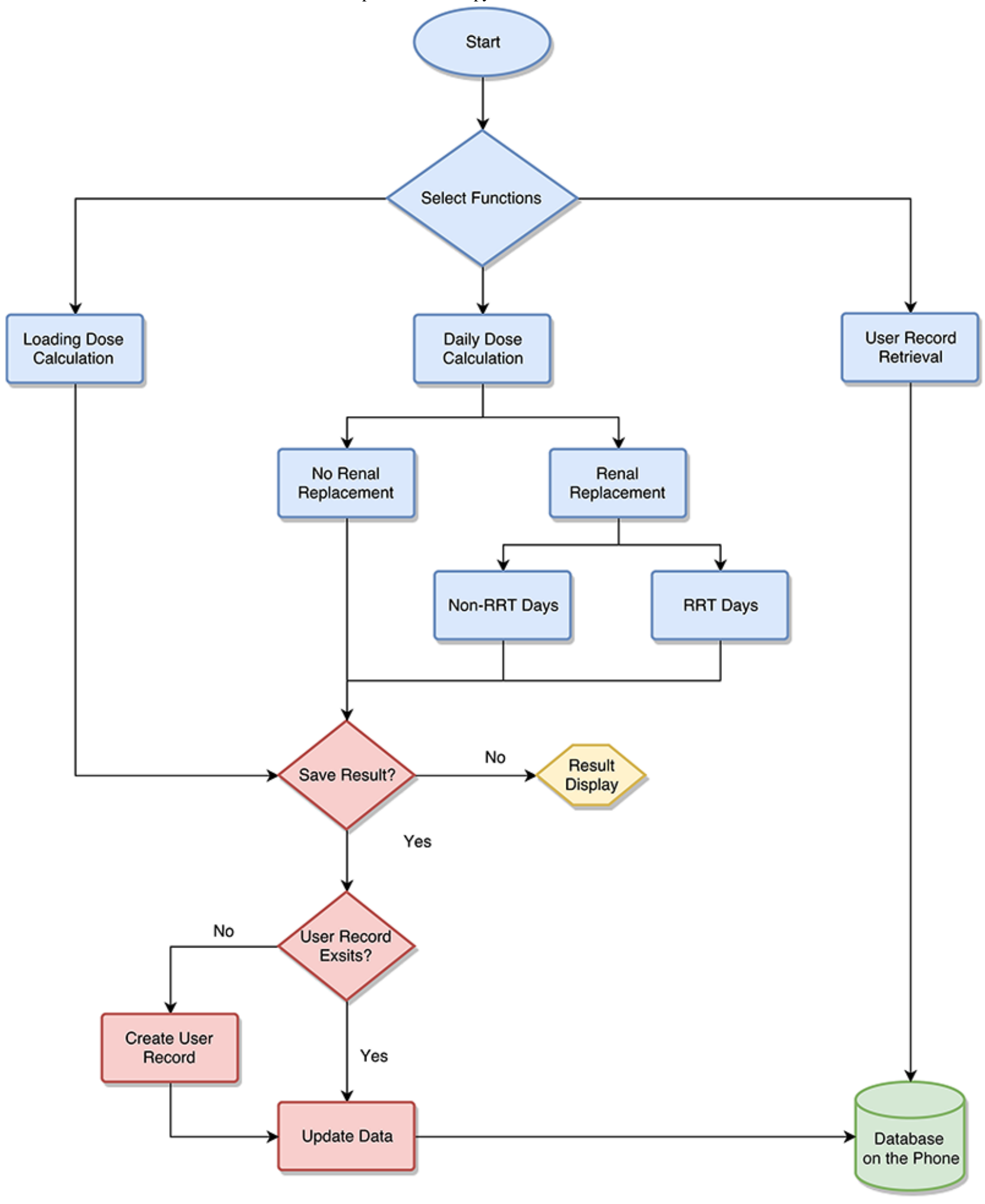

\section{PK/Pharmacodynamics (PD) Models of Intravenous Colistimethate in Patients for ColistinDose}

In 2017, we published dosing suggestions of intravenous colistimethate based on the largest population PK study conducted to date [11], including 214 critically ill patients (composed of 105 patients from an interim analysis [10] and 109 patients from an additional study site in Greece). In particular, the 2017 study included a larger number of patients with low creatinine clearances ( $\mathrm{CrCls})$, including 16 patients on hemodialysis, 4 on sustained low-efficiency dialysis (SLED), 7 on continuous veno-venous hemodialysis (CVVHD), and 2 on continuous veno-venous hemofiltration $(\mathrm{CVVH})$; these additional patients receiving RRT improved the ability to predict doses for these patients. The structural model from the interim analysis provided the starting point for further analysis, with all relevant covariates retested. After the new population PK analysis, the final model remained similar to the earlier model. 
For the calculation of the recommended loading dose (all patients), our final algorithm used ideal body weight (IBW) in all cases rather than the lower of actual body weight or IBW as per the interim analysis. The algorithm-derived maintenance (daily) dosing recommendations for patients with a $\mathrm{CrCl}>30$ $\mathrm{mL} / \mathrm{min}$ remained very similar to those proposed in the interim analysis. However, for patients with a $\mathrm{CrCl}<30 \mathrm{~mL} / \mathrm{min}$ (including patients prescribed intermittent hemodialysis [IHD] who were on a nondialysis day), the new recommendations were approximately $100 \%$ higher than those suggested in the interim analysis and also substantially higher than the doses currently approved by the Food and Drug Administration [23]. By utilizing the most recent equations, ColistinDose provides the most accurate dosing recommendations, especially for patients with low CrCls.

\section{Calculations of Dosage Regimens Using ColistinDose}

Calculations were based on the equations for the loading dose (all patients) and daily dose (for patients not receiving RRT and for patients on various types of RRT) developed in our recent population PK study [11]. Patients' IBW (kg) was calculated using the following equation: IBW $=50 \mathrm{~kg}$ ( $45.5 \mathrm{~kg}$ for females) $+2.3 \times$ number of inches over 5 feet (ie, 60 inches); $\mathrm{CrCl}$ ( $\mathrm{mL} / \mathrm{min}$ ) was calculated using the Cockcroft-Gault equation [24]. Three major functionalities were implemented in ColistinDose, namely calculations of the loading dose and maintenance daily dose, plus retrieval of historical records saved on the device. Recommended doses generated by ColistinDose were validated by comparison against doses calculated manually using the appropriate equations from the population PK study [11].

\section{Results}

\section{The ColistinDose App}

ColistinDose is simple to use and freely available for iOS (version 9 and above) at the Apple App Store. Upon opening the app to the main interface, the clinician is prompted to choose calculation of either a loading dose or a daily dose (Figure 2A). Prior to the publication of the final results of our population PK study [11], the importance of expediently achieving relatively high levels of formed colistin concentrations via a loading dose was already well known $[10,11,17,18]$. Following initiation of colistimethate treatment, the concentration of formed colistin increases slowly and may take up to $48 \mathrm{~h}$ before an acceptable average steady-state plasma concentration $\left(\mathrm{C}_{\mathrm{ss}, \mathrm{avg}}\right)$ of formed colistin is achieved. Achieving therapeutic concentrations rapidly in critically ill patients is important, as suboptimal PK/PD and delayed effective therapy are associated with increased mortality rates [25]. Low concentrations may also lead to development of resistance [26]. In ColistinDose, calculation of the loading dose is based on three key parameters: (1) the targeted $\mathrm{C}_{\mathrm{ss} \text {,avg }}$ of formed colistin, (2) gender (male/female), and (3) body height (inches or centimeters) (Figure 2A). Based on the recent population PK model, which found a colistin $\mathrm{C}_{\mathrm{ss}, \text { avg }}$ of $2 \mathrm{mg} / \mathrm{L}$ to be a suitable initial target concentration for intravenous treatment of bloodstream infections and some minor infections when the colistin minimal inhibitory concentration (MIC) was $\leq 2 \mathrm{mg} / \mathrm{L}$ [11], the selectable $\mathrm{C}_{\mathrm{ss}, \text { avg }}$ in ColistinDose has a default value of $2 \mathrm{mg} / \mathrm{L}$ and ranges from $0.5 \mathrm{mg} / \mathrm{L}$ to $4 \mathrm{mg} / \mathrm{L}$, with a step of $0.5 \mathrm{mg} / \mathrm{L}$. After pressing the "Calculate" button, the suggested loading dose is calculated using the following equation: $\mathrm{CBA}(\mathrm{mg})=\mathrm{C}_{\mathrm{ss}, \text { avg }}(\mathrm{mg} / \mathrm{L}) \times 2.0$ $\times$ IBW $(\mathrm{kg})$, where the IBW is calculated using the Devine formula described in "Methods" [27]. It is important to note that should the recommended loading dose exceed the maximum recommended daily dose of $300 \mathrm{mg}$ CBA (ie, 9 million IU) [23], a warning message (headed "Immediate Attention") will pop up to alert the user that the loading dose has been capped at $300 \mathrm{mg} \mathrm{CBA}$ due to safety considerations. Importantly, to help clinicians from different parts of the world, the calculated dose is expressed in both CBA and million IU to avoid prescribing errors that have been known to occur due to confusion over the conversion [19].

Because colistimethate (the administered prodrug) is predominantly renally cleared [28], whereas the formed colistin is largely cleared by nonrenal pathways [29], the equations for the calculation of the daily (maintenance) dose are complicated and require information on the renal function of the patient and whether they are on RRT [11]. Once calculation of the daily dose is selected from the main interface of ColistinDose, the $\mathrm{CrCl}(\mathrm{mL} / \mathrm{min})$ is determined via input of the patient's serum creatinine $(\mathrm{mg} / \mathrm{dL})$, gender (male/female), age (years), and weight (lbs or $\mathrm{kg}$ ). A screenshot of the daily dose calculation is shown in Figure 2A. The targeted $\mathrm{C}_{\mathrm{ss}, \text { avg }}(\mathrm{mg} / \mathrm{L})$ of formed colistin is also required [11]. Whether the patient is on RRT must also be selected. For patients not on RRT, the recommended daily dose is given in both CBA and million IU for 12-hourly administration (eg, $75 \mathrm{mg}$ CBA per $12 \mathrm{~h}$ ), with a suggestion that the first regular daily dose be administered 12 hours after the loading dose. This latter recommendation was taken from our population PK study [11]. For patients on RRT, three common dialysis options are provided: (1) IHD, (2) SLED, and (3) continuous RRT (CRRT). Based on our population PK analysis in patients receiving RRT, on a dialysis day of IHD, $20 \%$ or $50 \%$ of the baseline daily dose should be added after a 2-hour or 5-hour dialysis session, respectively [11]. For patients undergoing SLED or CRRT, 10\% of the baseline daily dose should be supplemented for every hour of dialysis [11]. When the particular type of dialysis is chosen and a daily dose calculated, the user is reminded of this information with a screen prompt. For example, if CRRT is selected, the following message will appear below the dosing recommendations: "During CRRT, add $10 \%$ per 1 hour of CRRT to the baseline daily dose," as suggested by our population PK study [11]. As per the loading dose, a warning message (headed "Immediate Attention") pops up to alert the user whenever the calculated daily dose exceeds the maximum recommended $300 \mathrm{mg}$ CBA (ie, 9 million IU) [11]. It should be noted that the actual calculated dose of colistimethate by ColistinDose can be higher, albeit with a cap of $300 \mathrm{mg}$. 
Figure 2. Screenshots illustrating the use of ColistinDose. (A) The main interface and calculations of the loading dose and daily dose. (B) Steps for saving the calculation results.
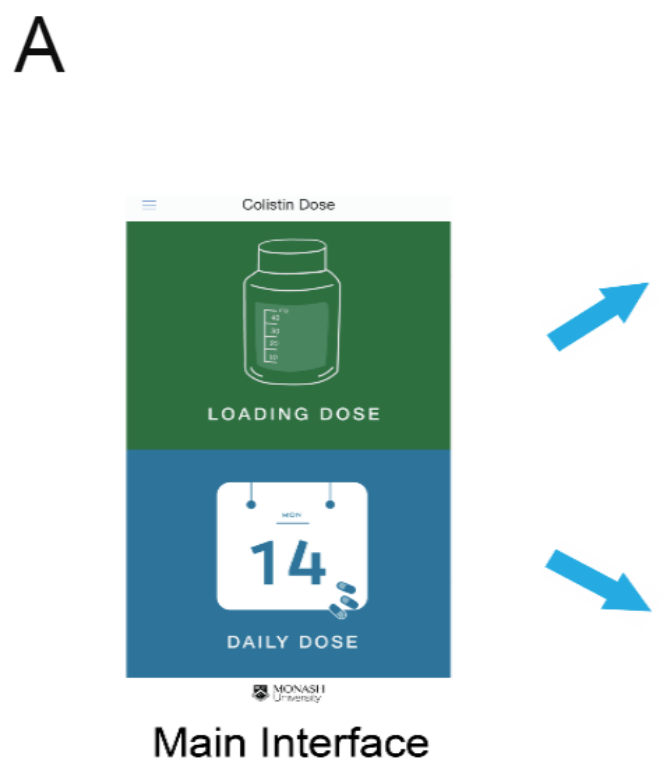
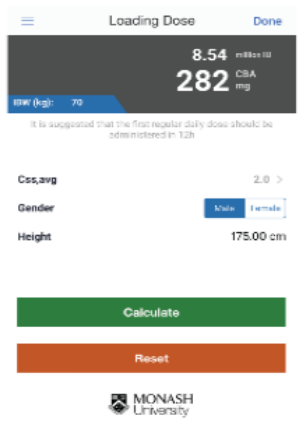

Loading Dose Calculation

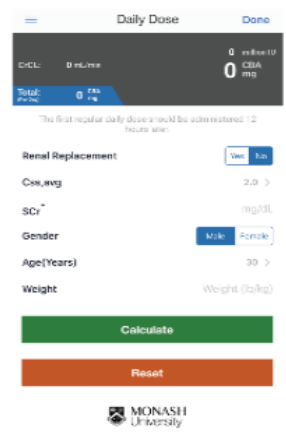

\section{Daily Dose Calculation}

\section{B}

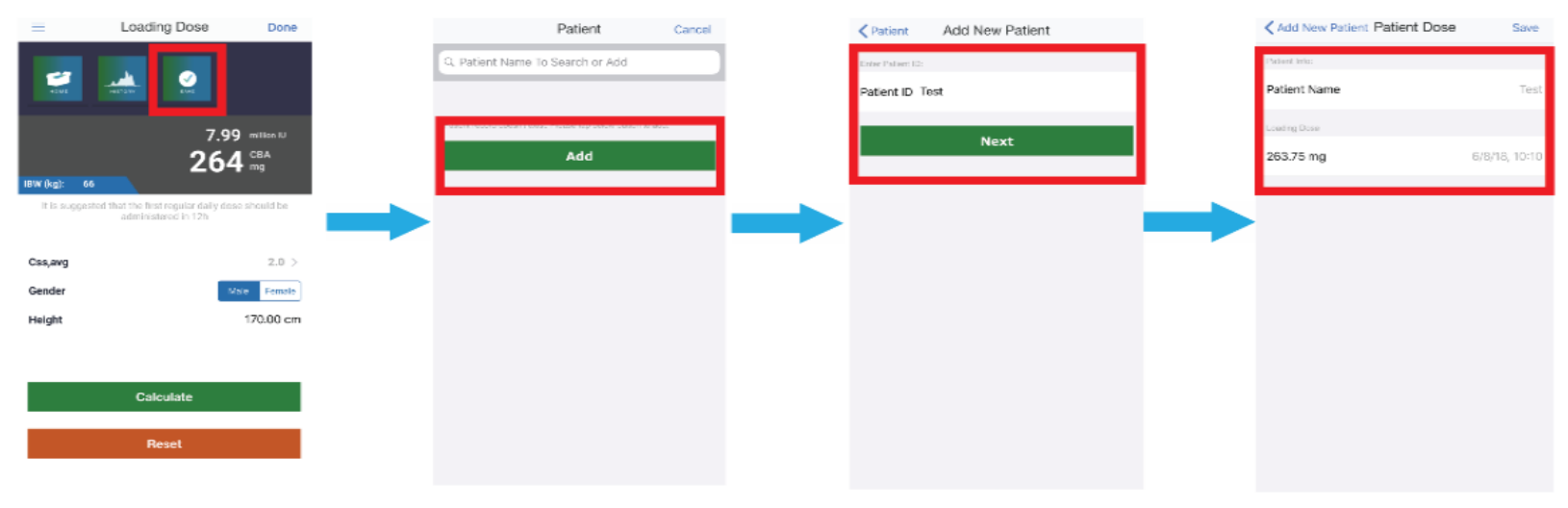

\section{Save Calculation Record}

Clinicians can choose to store the historical calculation records in the mobile device locally for each patient by using the "Save" option. Figure 2B demonstrates the saving of the dose calculations for a patient. With this function, clinicians can easily revisit previously calculated colistimethate doses as well as the types of renal dialysis, making dose adjustments where appropriate.

\section{Comparisons of Loading and Maintenance Daily Doses With Published Equations}

To evaluate the accuracy of ColistinDose against our published dosing algorithms, we performed mock calculations of loading and maintenance daily doses using the height, age, and body weight of 8 "patients," including 2 patients on each type of RRT (Multimedia Appendix 2). The calculated loading and daily doses for the 8 patients were almost identical to those calculated manually using the equations from the latest population PK model [11], the minor differences being due to number rounding. The rounded results are then converted to million IU. Overall, the results in Multimedia Appendix 2 demonstrate that the doses generated by ColistinDose accurately reflect doses recommended in patients with varying degrees of renal function and on different forms of RRT based on the most up-to-date published algorithms. In addition, coauthors JMP, IK, and KSK have evaluated and used ColistinDose in their clinical practices. The conversion function between two different dose definitions (ie, CBA versus IU) in ColistinDose provides a useful tool to prevent potential prescription errors. 


\section{Discussion}

\section{Comparison of ColistinDose With Existing Colistin Dosing Aids}

Compared with the two existing dosing aids (ie, colistin dosing calculator [21] and colistin calculator [22]), the advantages of ColistinDose are twofold. First, ColistinDose utilizes equations derived from the most recent and comprehensive population PK model published in 2017 [11], whereas the colistin dosing calculator and colistin calculator utilize dosing algorithms based on an interim population PK analysis published in 2011 [10]. Prior to the publication of the latest population PK study [11], we had published an interim population PK analysis of intravenous colistimethate that examined 105 critically ill patients across 3 study sites ( 2 in the United States and 1 in Thailand), including 12 patients on hemodialysis and 4 patients on CRRT (3 on CVVHD and 1 on CVVH) [10]. A nonlinear mixed-effects modeling tool, S-ADAPT, was used to analyze plasma concentration-versus-time data for both colistimethate and colistin and found that their dispositions were best described by linear 2- and 1-compartment models, respectively. These models then formed the basis for the development of colistimethate dosing suggestions for various categories of patients based on the PK data and its integration with PD data for Acinetobacter baumannii and Pseudomonas aeruginosa in murine thigh and lung infection models [12]. Prior to the interim population PK analysis [10], information on the PK of colistimethate and formed colistin was scarce and had been reported in only 32 patients, all with $\mathrm{CrCls}>40 \mathrm{~mL} / \mathrm{min}[18,30]$. As previously discussed, while dosing recommendations for patients with a $\mathrm{CrCl}>30 \mathrm{~mL} / \mathrm{min}$ were very similar between the interim and final population PK analyses, there were substantial differences for patients with lower $\mathrm{CrCls}$, including patients on dialysis; in the final analysis, recommendations for these latter patients were approximately $100 \%$ higher than those suggested in the interim analysis. Use of the interim dosing equations thus risks exposing patients to subtherapeutic concentrations of the active component, colistin, decreasing the likelihood of efficacy and increasing the possibility of the emergence of colistin resistance. As colistimethate is a last-line therapy often used in critically ill patients when no other therapeutic options are available, attaining sufficient plasma concentrations is imperative given the potential consequences of treatment failure.

Second, a major advantage of ColistinDose is that once the app is downloaded and installed, all calculations are conducted on the local mobile device (iPhone or iPad) without the need for an internet connection. In contrast, the colistin dosing calculator [21] and colistin calculator [22] are only available online.

\section{Patients' Privacy Protection}

To protect the patient's privacy and comply with ethics and data safety requirements, all data are only stored within and accessible from the app on the clinician's mobile device; deleting or uninstalling the app will simultaneously delete all associated information permanently, as demonstrated in the disclaimer and terms of use issued by Monash University (refer to Multimedia Appendix 3). Clinicians are only required to input the patient's ID — which could be either their patient ID number, a nickname, or anything easy to remember-to save the calculation results. Were the app to crash, only the crash data, not the patient's information or previously saved calculation results, would be sent to the app developer via the iOS under the users' agreement.

\section{Limitations and Outlook}

The primary limitation of ColistinDose is that the user interface is in English. This may cause inconvenience for non-English-native clinicians to use the app. In light of this, we are considering adding multiple languages in the next version of ColistinDose. Because colistin has a narrow therapeutic window and different renal functions can cause substantial interpatient variability of the PK [31], therapeutic drug monitoring is highly recommended to ensure favorable clinical outcomes in patients. Incorporation of new therapeutic drug monitoring results will improve the precision of our population PK model, as well as the future version of ColistinDose.

\section{Conclusions}

Colistimethate is increasingly used as a treatment of last resort in medically complex patients for infections caused by gram-negative "superbugs". However, its complicated pharmacology and confusing dose definition can cause patient safety issues. Here, we have developed an easy-to-use mobile app, ColistinDose, that can be used at the bedside to facilitate the calculation of intravenous colistimethate dosage regimens for adult patients with varying degrees of renal function. To date, ColistinDose has been downloaded from 53 countries and regions around the globe. The potential for ColistinDose to improve patient care is significant, and its easy-to-use interface and functionalities can significantly assist clinicians worldwide to reduce prescribing errors, maximize efficacy, and minimize emergence of resistance and the likelihood of acute kidney injury in patients.

\section{Acknowledgments}

We are grateful to Gabrielle Hirsch from The Office of the General Counsel, Monash University, for the legal assistance, disclaimer, and Terms of Use for ColistinDose. We also deeply appreciate the efforts of a number of staff from Monash University, including Moh-Lee Ng and Kevin Sweeney for risk assessment; Sharnie Hewlett and Vicki Goodwin for app design and logo branding; Mathew Radford, Thusitha Kumarasinghe, and the Monash eSolution Team for IT assessment, iTunes Connect account, and Monash Email management; and Joy Hewitt, John Morrison, Chacko Joseph, and the Monash Insurance Service Team for the app administration, release approval, and insurance services.

JL is supported by research grants from the National Institute of Allergy and Infectious Diseases of the National Institutes of Health (R01 AI132154 and AI146160). The content is solely the responsibility of the authors and does not necessarily represent 
the official views of the National Institute of Allergy and Infectious Diseases or the National Institutes of Health. CL was supported by the Bridging Postdoctoral Fellowship of Faculty of Medicine, Nursing and Health Sciences, Monash University (BPF17-0021), and is supported by a CJ Martin Early Career Research Fellowship of the National Health and Medical Research Council (NHMRC) of Australia (1143366). JL is an NHMRC Principal Research Fellow (1157909).

\section{Authors' Contributions}

JL conceived and designed the project; XH and CL designed, constructed, and tested the ColistinDose app; JMP, VSS, IK, KSK, BTT, PJB, YZ, and JS tested the ColistinDose app and provided critical feedback. CL, PJB, and JL drafted and revised the manuscript that has been read and approved by all the authors.

\section{Conflicts of Interest}

KSK is a consultant of Xellia Pharmaceuticals. The other authors have no conflicts to declare.

\section{Multimedia Appendix 1}

Detailed information of the open-source libraries and fonts utilized in ColistinDose.

[DOCX File, 14 KB-Multimedia Appendix 1]

\section{Multimedia Appendix 2}

Mock calculations of the maintenance daily doses in different patients.

[DOCX File, 19 KB-Multimedia Appendix 2]

\section{Multimedia Appendix 3}

Disclaimer and terms of use.

[DOCX File, 15 KB-Multimedia Appendix 3]

\section{References}

1. Jackson N, Czaplewski L, Piddock L. Discovery and development of new antibacterial drugs: learning from experience? J Antimicrob Chemother 2018;73(6):1452-1459. [doi: 10.1093/jac/dky019]

2. Luepke KH, Suda KJ, Boucher H, Russo RL, Bonney MW, Hunt TD, et al. Past, Present, and Future of Antibacterial Economics: Increasing Bacterial Resistance, Limited Antibiotic Pipeline, and Societal Implications. Pharmacotherapy 2017;37(1):71-84. [doi: 10.1002/phar.1868]

3. Boucher HW, Talbot GH, Benjamin DK, Bradley J, Guidos RJ, Jones RN, et al. 10 x '20 Progress--development of new drugs active against gram-negative bacilli: an update from the Infectious Diseases Society of America. Clinical Infectious Diseases 2013 Apr 17;56(12):1685-1694. [doi: 10.1093/cid/cit152]

4. Landman D, Georgescu C, Martin DA, Quale J. Polymyxins revisited. Clin Microbiol Rev 2008 Jul;21(3):449-465. [doi: $\underline{10.1128 / \mathrm{cmr} .00006-08]}$

5. Velkov T, Roberts KD, Thompson PE, Li J. Polymyxins: a new hope in combating Gram-negative superbugs? Future Med Chem 2016 Jun;8(10):1017-1025. [doi: 10.4155/fmc-2016-0091]

6. Bergen PJ, Li J, Rayner CR, Nation RL. Colistin methanesulfonate is an inactive prodrug of colistin against Pseudomonas aeruginosa. Antimicrob Agents Chemother 2006 Jun;50(6):1953-1958. [doi: 10.1128/aac.00035-06]

7. Azad MAK, Nation RL, Velkov T, Li J. Mechanisms of polymyxin-induced nephrotoxicity. Adv Exp Med Biol 2019;1145:305-319. [doi: 10.1007/978-3-030-16373-0_18]

8. Kubin CJ, Ellman TM, Phadke V, Haynes LJ, Calfee DP, Yin MT. Incidence and predictors of acute kidney injury associated with intravenous polymyxin B therapy. J Infect 2012 Jul;65(1):80-87. [doi: 10.1016/j.jinf.2012.01.015]

9. Rigatto MH, Behle TF, Falci DR, Freitas T, Lopes NT, Nunes M, et al. Risk factors for acute kidney injury (AKI) in patients treated with polymyxin B and influence of AKI on mortality: a multicentre prospective cohort study. J Antimicrob Chemother 2015 Jan 20;70(5):1552-1557. [doi: 10.1093/jac/dku561]

10. Garonzik SM, Li J, Thamlikitkul V, Paterson DL, Shoham S, Jacob J, et al. Population pharmacokinetics of colistin methanesulfonate and formed colistin in critically ill patients from a multicenter study provide dosing suggestions for various categories of patients. Antimicrob Agents Chemother 2011 May 09;55(7):3284-3294. [doi: 10.1128/aac.01733-10]

11. Nation RL, Garonzik SM, Thamlikitkul V, Giamarellos-Bourboulis EJ, Forrest A, Paterson DL, et al. Dosing guidance for intravenous colistin in critically-ill patients. Clin Infect Dis 2017;64(5):565-571. [doi: 10.1093/cid/ciw839]

12. Cheah S, Wang J, Nguyen VTT, Turnidge JD, Li J, Nation RL. New pharmacokinetic/pharmacodynamic studies of systemically administered colistin against Pseudomonas aeruginosa and Acinetobacter baumannii in mouse thigh and lung infection models: smaller response in lung infection. J Antimicrob Chemother 2015 Aug 27;70(12):3291-3297. [doi: $\underline{10.1093 / \mathrm{jac} / \mathrm{dkv} 267]}$ 
13. Grégoire N, Mimoz O, Mégarbane B, Comets E, Chatelier D, Lasocki S, et al. New colistin population pharmacokinetic data in critically ill patients suggesting an alternative loading dose rationale. Antimicrob Agents Chemother 2014 Sep 29;58(12):7324-7330. [doi: 10.1128/aac.03508-14]

14. Jacobs M, Grégoire N, Mégarbane B, Gobin P, Balayn D, Marchand S, et al. Population pharmacokinetics of colistin methanesulfonate and colistin in critically ill patients with acute renal failure requiring intermittent hemodialysis. Antimicrob Agents Chemother 2016 Jan 04;60(3):1788-1793. [doi: 10.1128/aac.01868-15]

15. Karaiskos I, Friberg LE, Pontikis K, Ioannidis K, Tsagkari V, Galani L, et al. Colistin population pharmacokinetics after application of a loading dose of $9 \mathrm{MU}$ colistin methanesulfonate in critically ill patients. Antimicrob Agents Chemother 2015 Sep 14;59(12):7240-7248. [doi: 10.1128/aac.00554-15]

16. Bergen PJ, Bulitta JB, Forrest A, Tsuji BT, Li J, Nation RL. Pharmacokinetic/pharmacodynamic investigation of colistin against Pseudomonas aeruginosa using an in vitro model. Antimicrob Agents Chemother 2010 Sep;54(9):3783-3789. [doi: $\underline{10.1128 / \text { aac.00903-09] }}$

17. Mohamed AF, Karaiskos I, Plachouras D, Karvanen M, Pontikis K, Jansson B, et al. Application of a loading dose of colistin methanesulfonate in critically ill patients: population pharmacokinetics, protein binding, and prediction of bacterial kill. Antimicrob Agents Chemother 2012 May 21;56(8):4241-4249. [doi: 10.1128/aac.06426-11]

18. Plachouras D, Karvanen M, Friberg LE, Papadomichelakis E, Antoniadou A, Tsangaris I, et al. Population pharmacokinetic analysis of colistin methanesulfonate and colistin after intravenous administration in critically ill patients with infections caused by gram-negative bacteria. Antimicrob Agents Chemother 2009 Aug;53(8):3430-3436. [doi: 10.1128/aac.01361-08]

19. Li J, Coulthard K, Nation R. Labelling conventions and product package insert of parenteral polymyxins: factors causing potential medication errors and impeding optimal clinical use. Adv Exp Med Biol 2019;1445:133-141. [doi: 10.1007/978-3-030-16373-0 10]

20. Ventola CL. Mobile devices and apps for health care professionals: uses and benefits. P T 2014 May;39(5):356-364. [Medline: 24883008]

21. McAuley D. Colistin dosing calculator (Beta). GlobalRPh. URL: https://globalrph.com/medcalcs/ colistin-dosing-calculator-beta/ [accessed 2020-09-23]

22. Justo JA. Colistin calculator. ClinCalc. URL: https://clincalc.com/Colistin/ [accessed 2020-09-23]

23. U.S. Food and Drug Administration. Coly-Mycin M Parenteral (Colistmethate for Injection, USP). U.S. Food and Drug Administration.: U.S. Food and Drug Administration URL: https://www.accessdata.fda.gov/drugsatfda_docs/label/2017/ 050108s033lbl.pdf [accessed 2020-08-23]

24. Cockcroft DW, Gault H. Prediction of creatinine clearance from serum creatinine. Nephron 1976;16(1):31-41. [doi: 10.1159/000180580]

25. Raghavan M, Marik PE. Management of sepsis during the early "golden hours". J Emerg Med 2006 Aug;31(2):185-199. [doi: 10.1016/j.jemermed.2006.05.008]

26. Lim LM, Ly N, Anderson D, Yang JC, Macander L, Jarkowski A, et al. Resurgence of colistin: a review of resistance, toxicity, pharmacodynamics, and dosing. Pharmacotherapy 2010 Dec;30(12):1279-1291. [doi: 10.1592/phco.30.12.1279]

27. Devine BJ. Gentamicin therapy. Drug Intell Clin Pharm 1974;8:650-655.

28. Li J, Milne RW, Nation RL, Turnidge JD, Smeaton TC, Coulthard K. Pharmacokinetics of colistin methanesulphonate and colistin in rats following an intravenous dose of colistin methanesulphonate. J Antimicrob Chemother 2004 Mar 24;53(5):837-840. [doi: $10.1093 / \mathrm{jac} / \mathrm{dkh} 167]$

29. Li J, Milne RW, Nation RL, Turnidge JD, Smeaton TC, Coulthard K. Use of high-performance liquid chromatography to study the pharmacokinetics of colistin sulfate in rats following intravenous administration. Antimicrob Agents Chemother 2003 May;47(5):1766-1770. [doi: 10.1128/aac.47.5.1766-1770.2003]

30. Markou N, Markantonis SL, Dimitrakis E, Panidis D, Boutzouka E, Karatzas S, et al. Colistin serum concentrations after intravenous administration in critically ill patients with serious multidrug-resistant, gram-negative bacilli infections: A prospective, open-label, uncontrolled study. Clin Ther 2008 Jan;30(1):143-151. [doi: 10.1016/j.clinthera.2008.01.015]

31. Tsuji BT, Pogue JM, Zavascki AP, Paul M, Daikos GL, Forrest A, et al. International consensus guidelines for the optimal use of the polymyxins: endorsed by the American College of Clinical Pharmacy (ACCP), European Society of Clinical Microbiology and Infectious Diseases (ESCMID), Infectious Diseases Society of America (IDSA), International Society for Anti - infective Pharmacology (ISAP), Society of Critical Care Medicine (SCCM), and Society of Infectious Diseases Pharmacists (SIDP). Pharmacotherapy 2019 Feb 02;39(1):10-39. [doi: 10.1002/phar.2209]

\section{Abbreviations}

CBA: colistin base activity

CMS: colistin methanesulfonate

CrCl: creatinine clearance

CRRT: continuous renal replacement therapy

Css,avg: average steady-state plasma colistin concentration

CVVH: continuous veno-venous hemofiltration 
CVVHD: continuous veno-venous hemodialysis

IBW: ideal body weight

IHD: intermittent hemodialysis

IU: international units

MIC: minimal inhibitory concentration

PD: pharmacodynamics

PK: pharmacokinetics

RRT: renal replacement therapy

SLED: sustained low-efficiency dialysis

Edited by G Eysenbach, L Buis; submitted 21.05.20; peer-reviewed by M Scheetz, KL Mauco; comments to author 02.08.20; revised version received 23.09.20; accepted 28.10.20; published 16.12.20

Please cite as:

Hua X, Li C, Pogue JM, Sharma VS, Karaiskos I, Kaye KS, Tsuji BT, Bergen PJ, Zhu Y, Song J, Li J

ColistinDose, a Mobile App for Determining Intravenous Dosage Regimens of Colistimethate in Critically Ill Adult Patients:

Clinician-Centered Design and Development Study

JMIR Mhealth Uhealth 2020;8(12):e20525

URL: http://mhealth.jmir.org/2020/12/e20525/

doi: $\underline{10.2196 / 20525}$

PMID: 33325835

(CXueliang Hua, Chen Li, Jason M Pogue, Varun S Sharma, Ilias Karaiskos, Keith S Kaye, Brian T Tsuji, Phillip J Bergen, Yan Zhu, Jiangning Song, Jian Li. Originally published in JMIR mHealth and uHealth (http://mhealth.jmir.org), 16.12.2020. This is an open-access article distributed under the terms of the Creative Commons Attribution License (https://creativecommons.org/licenses/by/4.0/), which permits unrestricted use, distribution, and reproduction in any medium, provided the original work, first published in JMIR mHealth and uHealth, is properly cited. The complete bibliographic information, a link to the original publication on http://mhealth.jmir.org/, as well as this copyright and license information must be included. 